\title{
Subjetividad, determinabilidad y reciprocidad en Maimon y Fichte
}

\section{Gonzalo Santaya}

\section{(2) OpenEdition \\ Journals}

Edición electrónica

URL: http://journals.openedition.org/ref/770

ISSN: 2258-014X

Editor

EuroPhilosophie Editions

Referencia electrónica

Gonzalo Santaya, "Subjetividad, determinabilidad y reciprocidad en Maimon y Fichte », Revista de Estud(i)os sobre Fichte [En línea], 15 | 2017, Publicado el 01 diciembre 2017, consultado el 31 diciembre 2017. URL : http://journals.openedition.org/ref/770

Este documento fue generado automáticamente el 31 diciembre 2017.

(c) EuroPhilosophie 


\title{
Subjetividad, determinabilidad y reciprocidad en Maimon y Fichte
}

\author{
Gonzalo Santaya
}

1 Salomon Maimon y Johann Gottlieb Fichte comparten más que una época y un interés profundo por la filosofía kantiana. Comparten el haber transitado vidas tortuosas, llenas de obstáculos, pobreza, injurias e indiferencia. Todas estas dificultades se articulan en el caso de ambos pensadores en torno a un mismo motivo: la filosofía como sentido de la existencia. Comparten también un cierto parecer sobre el diagnóstico de su época y la tarea que la misma impone: a sus ojos, Kant había colocado a la filosofía en una vía completamente nueva, extremadamente potente, pero el modo en que el filósofo de Königsberg condujo su empresa no derivó inmediatamente en feliz término, y la recepción de la intelectualidad contemporánea no parecía acercarse a nada superador. Estos dos kantianos renegados se harán, cada uno a su modo, responsables de una reformulación de la filosofía trascendental. Pero a pesar de los incesantes esfuerzos que acompañaron sus proyectos vitales y filosóficos -difícil separar uno de otro en ambos casos-, éstos se hallan atravesados por una permanente y desigual discusión con sus entornos intelectuales, políticos y religiosos, y la muerte encontrará a ambos tempranamente en soledad y abandono. Con todo, los dos pensadores juegan un papel esencial en el nacimiento de lo que suele llamarse Idealismo Alemán, tanto por las exigencias que plantearon a la filosofía kantiana como por sus intentos de responder a estas exigencias.

2 Existen fuertes resonancias entre el Ensayo de filosofía trascendental de Maimon (de aquí en más, EFT), aparecido en 1790, y el Fundamento de toda la doctrina de la ciencia de Fichte (de aquí en más FDC), de 1794/95. Si bien esta afinidad raramente está plasmada explícitamente en la letra, existen indicios que muestran que ambos pensadores estaban al tanto de los pasos que el otro daba en el terreno filosófico. A este respecto es sumamente ilustrativo un pasaje de una carta de Fichte a Reinhold del año 1795:

Frente al talento de Maimon, mi respeto no tiene límites. Creo firmemente, y estoy dispuesto a demostrarlo, que por él la filosofía kantiana en su totalidad, tanto en general como en la forma en la que usted la entendió, ha sido derribada desde su fundamento. Él ha hecho todo esto sin que nadie se dé cuenta. ${ }^{1}$ 
Este "respeto ilimitado" de Fichte hacia Maimon está también sugerido en el FDC, donde el filósofo judío aparece mencionado como "uno de los más grandes pensadores de nuestro tiempo". ${ }^{2}$ Este tipo de referencias en un autor que no se destaca por regalar elogios sugieren que Fichte conocía bien la obra de Maimon, y que en el período de gestación de la primera exposición sistemática y completa de su pensamiento (el FDC), el EFT (aparecido cuatro años antes) pudo haber jugado un importante papel (así como, recíprocamente, se ha sostenido que los escritos del joven Fichte sobre la revolución francesa jugaron un papel en las incursiones maimonianas en el derecho natural). ${ }^{3}$

$4 \quad$ El objetivo principal de estas páginas es analizar algunos aspectos que tornan plausible la hipótesis de una posible influencia implícita del EFT sobre el FDC. Se argumentará que Fichte elabora su teoría como un reconocimiento de la importancia del planteamiento de Maimon y una reformulación de algunos de sus conceptos principales, con vistas a una refutación de su escepticismo. Se examinarán tres nociones centrales de estas obras: la estructura de la subjetividad (escindida en ambos autores en un conflicto entre finitud e infinitud), la ley de determinabilidad (que constituye para Maimon el principio supremo de la cognición pura, pero que a la vez, dada la estructura escindida de la subjetividad, lo conduce finalmente a sus conclusiones escépticas), y el concepto de determinación recíproca (el hilo a través del cual Fichte entrelaza el recorrido argumental del FDC, y a la vez da respuesta al desafío escéptico maimoniano, por cuanto permite superar el conflicto de la subjetividad escindida). Se intentará, por último, mostrar que Maimon, por permanecer en el punto de vista de la razón cognoscitiva, cae en un escepticismo fundado en una división entre lo finito y lo infinito; división que Fichte se propone conciliar.

\section{Maimon, determinabilidad y razón escéptica}

5 En un artículo publicado el mismo año que el EFT, Maimon presenta una crítica a la filosofía kantiana que, si se permite el anacronismo, suena -en principio- completamente fichteana: el sistema de Kant es por sí mismo "insuficiente para prevenir todo dogmatismo venidero". ${ }^{4}$ La semilla de este dogmatismo (plantada en la Crítica para la posteridad) está en la noción de "cosa en sí", que Maimon redefine:

De acuerdo con Kant, la cosa en sí es aquello a lo que el concepto o la representación se refiere por fuera de nuestro poder cognoscitivo. Yo, en cambio, afirmo que la cosa en sí entendida de este modo es un sinsentido porque somos incapaces de probar su existencia o de formarnos un concepto de ella. Para mí, las cosas en sí mismas y los conceptos o representaciones de cosas son objetivamente una y la misma cosa: difieren sólo subjetivamente, es decir, en relación a la completitud de nuestro conocimiento. [...] La cosa en sí es una idea de la razón, dada por la razón misma, para resolver una antinomia universal del pensamiento en general. Pues éste comprende la relación de una forma (regla del entendimiento) a una materia (lo dado subsumido bajo ella). [...] [L]o requerido para la completitud del pensamiento de un objeto es que nada sea dado y todo pensado. No podemos rechazar ninguno de estos dos requerimientos como ilegítimo, sino que debemos satisfacer ambos haciendo nuestro conocimiento cada vez más completo, un proceso en el cual la materia se aproxima a la forma al infinito, y esto resuelve la antinomia. ${ }^{5}$

6 Para ilustrar esto, Maimon apela a un procedimiento matemático: el método exhaustivo. Según esta analogía (ver la figura a continuación), un polígono regular es un concepto en relación al círculo con respecto al cual podemos pensar al primero como inscripto o circunscripto en el segundo; este círculo, a su vez, debe considerarse como la cosa en sí con 
respecto al polígono. La tarea del pensamiento, que desea conocer el círculo pero conoce sólo el polígono, es seguir una regla de producción de aquél, multiplicando al infinito los lados del polígono. En el límite, siguiendo esta regla, el polígono de infinitos lados y el círculo coinciden plenamente. El círculo completa materialmente la regla formal del entendimiento que manda añadir sucesivamente lados al polígono. Esta analogía pretende definir el pensamiento atenuando la radical diferencia de naturaleza que Kant postulaba entre materia y forma de la cognición. Aquí ya no hay un afuera para nuestras facultades cognoscitivas, sino una finitud constitutiva del pensamiento humano que lo condena a una tarea de aproximación infinita a la cognición completa.

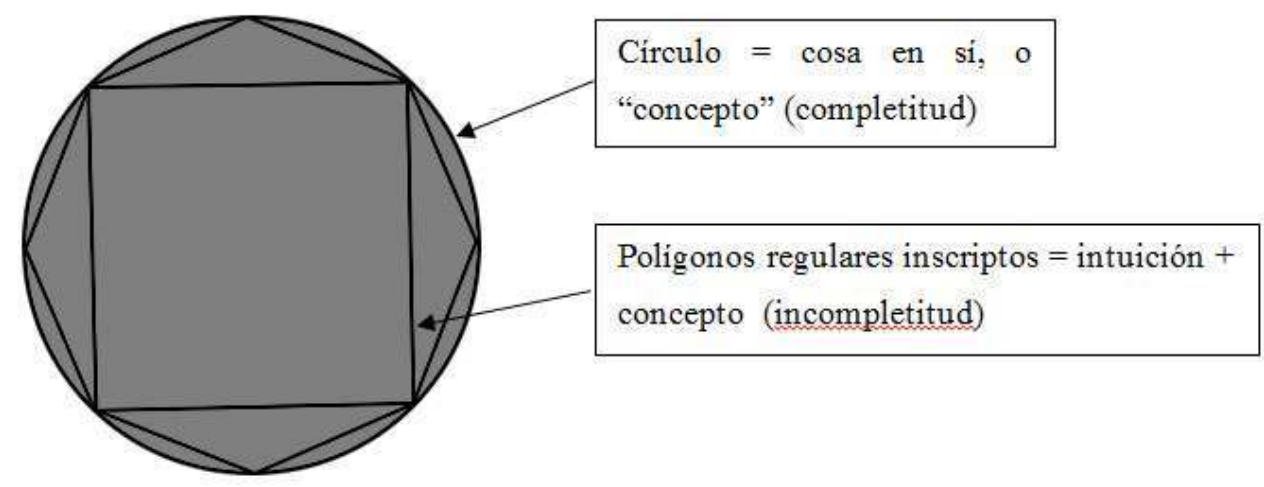

7 Por lo tanto, la crítica a la exterioridad de la cosa en sí de Kant se traslada al interior de su doctrina sobre la subjetividad, es decir, se vuelve una crítica al modo en que concibe las facultades cognoscitivas. Esta crítica se desarrolla extensamente en el EFT en ocasión del tratamiento de la cuestión quid juris, a saber: ¿cómo es posible que formas a priori deban aplicarse a cosas dadas a posteriori? Maimon advierte que este problema permanece insoluble en tanto y en cuanto no se muestre un fundamento de unificación de lo dado y lo pensado, y cree poder mostrar ese fundamento por medio de una recuperación del modelo de la metafísica prekantiana, interpretada, por cierto, a través del tamiz de la Crítica:

En el sistema kantiano, donde la sensibilidad y el entendimiento son dos fuentes de cognición totalmente diferentes, esta pregunta [sc. quid juris] es, como he mostrado, insoluble. Por otro lado, en el sistema de Leibniz-Wolff, ambos fluyen desde una y la misma fuente cognoscitiva (la diferencia yace en el grado de completitud de esta cognición), y así la pregunta se responde fácilmente. [...] Asumimos un entendimiento infinito (al menos como idea), para el cual las formas sean al mismo tiempo objetos del pensamiento, o que produzca desde sí mismo todos los modos posibles de relaciones y conexiones entre las cosas (las ideas). Nuestro entendimiento es lo mismo, pero de modo limitado. ${ }^{6}$

La heterogeneidad entre sensibilidad y entendimiento ya no radica en una diferencia de naturaleza, como en Kant, ${ }^{7}$ sino de grado: ésta será la matriz de la doctrina maimoniana de la subjetividad, escindida según el modelo de un entendimiento infinito y sus modos, los entendimientos finitos. La génesis de la experiencia se da entonces no ya en el encuentro entre condiciones extrínsecas, sino en la inmanencia de una infinitud que el pensamiento finito no puede reflejar sino parcialmente, emplazado en el punto de vista de una conciencia determinada.

9 La perspectiva de la finitud presupone una pérdida en la unicidad de la inmanencia. Si bien no hay nada exterior para el poder cognoscitivo del entendimiento, el sujeto finito paga el precio de su limitación recibiendo, sin producirlos por sí mismo, datos sensibles. 
Pero dada la exigencia de completitud de la cognición, lo que la sensibilidad recibe y dona al entendimiento (finito) debe ser a su vez un producto del entendimiento (infinito): se trata de las ideas del entendimiento, también llamadas diferenciales, tomadas del método leibniziano para el cálculo infinitesimal. Estos diferenciales se asocian a las reglas del entendimiento para la generación de los objetos de la intuición. Carentes por sí mismos de manifestación en la intuición, la determinan, sin embargo, a partir de sus relaciones:

Estos diferenciales de objetos son los llamados noumena; pero los objetos mismos que emergen de ellos son phaenomena. Con respecto a la intuición $=0$, el diferencial de cada objeto tal es $d x=0, d y=0$, etc.; $\sin$ embargo, sus relaciones no son $=0$, sino que pueden ser dadas como determinadas en las intuiciones que surgen de ellos. ${ }^{8}$

10 En el seno del entendimiento infinito, cada diferencial relaciona con los demás mediante reglas, resultando en la producción de objetos del pensamiento puro, modos de generación y subsunción de una multiplicidad bajo la unidad de la regla. Mientras que en el seno del entendimiento finito, las diferenciales se corresponden con la intuición de un objeto espacio-temporal. Dado que ninguna determinación espacio-temporal se capta en sí misma, sino en relación a otras determinaciones, ${ }^{9}$ la determinación de una intuición depende de sus relaciones con otras intuiciones, y éstas, a su vez, de las relaciones de los diferenciales entre sí. De ahí la completitud del entendimiento infinito. "[P]ara un entendimiento infinito", afirma Maimon, "todo está en sí mismo completamente determinado porque éste piensa todas las relaciones reales posibles entre las ideas como sus principios". ${ }^{10}$ Así como la cosa en sí es de la misma naturaleza que la representación, pero llevando a ésta al infinito, el entendimiento infinito es de la misma naturaleza que el finito, pero mientras que el primero es capaz de pensar la cosa en sí, dado que accede a la totalidad infinita de sus determinaciones, el segundo sólo concibe de modo parcial algunas determinaciones, desligadas de su regla de producción. El entendimiento es una facultad activa y productiva según reglas, pero en su versión degradada (finita), esta facultad no encuentra en sí misma la regla de producción de sus objetos, sino que los recibe como si no fuera la fuente de la totalidad de sus determinaciones.

11 El modo de operar del entendimiento infinito se explica desde una ley que Maimon presenta en su EFT: la ley de la determinabilidad [Bestimmbarkeit]. ${ }^{11} \mathrm{~A}$ través de ella Maimon describe la naturaleza del pensamiento real, ya no del posible o meramente formal -a cuya elucidación Kant se había dedicado exclusivamente. De acuerdo con Maimon, desde el punto de vista finito todo pensamiento determinante de un objeto, o pensamiento real, es sintético. Existen básicamente dos tipos de síntesis, productoras de dos tipos de conceptos.

Una síntesis es sólo posible si una de sus partes constitutivas no puede ser pensada sin la otra. Esto puede darse unilateralmente -como en la síntesis de sujeto y predicado (determinable y determinación) de un concepto absoluto-, o recíprocamente, como en la síntesis de los correlatos de un concepto relacional. ${ }^{12}$

12 Es el primer tipo de síntesis aquí descripto el que cumple las condiciones de la ley de determinabilidad. Para un entendimiento finito, esta ley puede ejemplificarse mediante construcciones matemáticas: puedo pensar la síntesis "triángulo" sin pensar "equilátero" o "isósceles", etc. -pensar lo determinable sin la determinación-, y, luego, ir construyendo o deduciendo sucesivamente determinaciones a partir de lo determinable, produciendo el objeto y su conocimiento de manera simultánea y necesaria (dado que la cosa en sí y su representación difieren sólo en grado, podemos decir que esta ley es simultáneamente ley del ser y del pensar). Este tipo de síntesis, que da por resultado un 
“concepto absoluto", es la ley suprema del pensamiento sintético, y principio de la lógica trascendental.

13 El segundo tipo de síntesis (la recíproca) puede ejemplificarse con conceptos relacionales como "causalidad" u "oposición", cuyos elementos (respectivamente: causa y efecto, realidad y negación) son sólo concebibles en y por su mutua relación. En este caso, lo producido es una relación, no un objeto. Al aplicarlos, el entendimiento finito corre el riesgo de realizar una síntesis arbitraria, no fundada en una regla de producción. El escepticismo de Maimon resulta de que, enfrentados a la experiencia, sólo poseemos conceptos relacionales para subsumir lo dado. El caso de la causalidad es paradigmático: la lógica trascendental de Kant no permitiría dar una respuesta a la crítica humeana a ese concepto, pues la necesidad subjetiva que ponemos en la aplicación de dicha categoría no implica necesidad objetiva. Ésta sería sólo alcanzable para un entendimiento infinito, que piensa simultáneamente todas las reglas de producción y todas sus relaciones, pudiendo distinguir en cada caso la conexión necesaria (intelectiva) de la yuxtaposición arbitraria (intuitiva). La filosofía queda así indefensa ante la pregunta quid facti?, con lo que la necesidad de los juicios de experiencia permanecerá siempre problemática. Tenemos entonces:

una asociación de percepciones, no un juicio del entendimiento [...]; en consecuencia, no hay proposiciones de experiencia propiamente hablando (que expresen necesidad) [...]. De aquí que la lógica es incapaz de dar una marca distintiva confiable sobre la realidad de sus formas. ${ }^{13}$

Todas nuestras categorías son conceptos relacionales que deben recibir su materia de otra parte. Por ejemplo, gracias a la categoría de sustancia-accidente, puedo emitir el juicio "el oro es amarillo", porque de ese modo me es dado este objeto espacio-temporal particular, y no porque pueda poner el sujeto "oro" como determinable, bajo una regla de generación de la cual pueda extraer necesariamente el predicado "amarillo". Incluso en el conocimiento matemático nuestra cognición no es pura (es decir, exclusivamente intelectiva), por estar atada a la intuición (que no por ser a priori deja de ser la manifestación de un entendimiento incompleto). En consecuencia, el ser humano está vedado en sus posibilidades de acceso al conocimiento de lo real, condenado a continuar al infinito su tarea cognoscitiva, aproximándose continuamente a la cosa en sí. Condena que, a pesar de todo, el espíritu lleva adelante como su más esencial, elevada y deseada

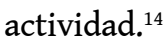

\section{Fichte, determinación recíproca y razón práctica}

15 Podemos concebir la empresa fichteana del FDC como la elaboración de una interpretación de Kant que -al igual que el Kant de la primera Crítica- lucha con una mano contra el fatalismo dogmático, y con la otra contra el escéptico. ${ }^{15}$ En este sentido, Maimon tiene una doble importancia: no sólo es un "escéptico trascendental" a refutar, también ha trastocado la manera de entender a Kant, señalando puntos en los cuales el esquema kantiano debe revisarse; en particular, la insuficiencia de la letra kantiana para liquidar al dogmatismo metafísico (con su negación de la existencia autónoma de una cosa en sí), y el cuestionamiento de la heterogeneidad entendimiento-sensibilidad. A esta agenda, Fichte suma su propia preocupación por una sistematicidad carente en la exposición kantiana, reelaborando toda la filosofía trascendental a partir de un principio supremo del saber humano: el Yo. 
16 Es importante señalar el rechazo fichteano a la concepción maimoniana del progreso infinito en la determinación. Que Fichte no postula esta especie de progresión se ve en su crítica al concepto de infinito matemático, tal como puede encontrarse en su Fundamento del derecho natural según los principios de la doctrina de la ciencia. Allí, Fichte declara incomprensible el método exhaustivo (aquél procedimiento geométrico que Maimon ponía como ejemplo de la progresión infinita):

¿Es acaso el concepto de una tarea de continuar dividiendo al infinito los lados del polígono, por consiguiente, la tarea de un determinar infinito? [...] Si continuáis dividiendo al infinito, como debéis según la tarea, no llegáis nunca a medir. Pero si queréis medir, tenéis que haber dejado de dividir. ${ }^{16}$

Esta idea se repite en una nota en Sobre el concepto de la doctrina de la ciencia, donde Fichte afirma que ante esta tarea infinita "nuestra limitada imaginación tiene que poner un punto terminal", ${ }^{17}$ y aclara que en el contexto de este procedimiento, que implica la determinación del espacio, "no estamos ya en el dominio de la doctrina de la ciencia, sino en el terreno de una ciencia particular llamada geometría [...] rigurosamente separada de la doctrina de la ciencia". ${ }^{18}$ En este punto en particular, podríamos decir que Fichte se apega más fielmente a la concepción kantiana, dada la crítica que Kant dirige -en la "Doctrina trascendental del método" de la Crítica de la razón pura- a la aplicación ilegítima del método matemático a la filosofía. ${ }^{19}$ A diferencia del modelo maimoniano de la progresión infinita, extrapolado de la geometría, el Yo fichteano seguirá, como veremos, el de la continuación indefinida de la determinación. (Este punto nos parece también relevante para alejar a Fichte de lo que más adelante se llamará "mala infinitud".)

Al poner a la cosa en sí como límite de un proceso infinito de conocimiento a la manera del método exhaustivo (y por lo tanto, como algo ya dado en cierto modo, pues la operación sobre el polígono ya presupone el círculo), Maimon prefigura o predetermina una dirección para la actividad intelectiva. Esta actividad va hacia el infinito en tanto debe repetir sin término una misma operación (añadir lados). La propuesta fichteana, en cambio, dará a la libertad un rol fundamental, en la medida en que no se basa en la capacidad de seguir permanentemente una dirección preestablecida, sino en la capacidad de trazar por sí misma una dirección, que luego podrá continuarse indefinidamente. La continuación indefinida, como alternativa a la aproximación infinita, deviene en este punto el índice de una primacía de la libertad en la caracterización de la razón humana. Esto vendrá acompañado, como veremos a continuación, de una diferenciación en los papeles de los elementos de la lógica trascendental, mediante una nueva caracterización de la noción de reciprocidad.

19 El Yo fichteano puede equipararse al entendimiento maimoniano en la medida en que en ambos encontramos una subjetividad productora de su objeto, inmanente y fundada en sí misma, escindida en un conflicto entre finitud e infinitud. Sin embargo, Fichte eleva esta subjetividad a nivel de primer principio, definiéndola de tal modo que pueda sentar las condiciones para superar dicho conflicto. Si la actividad del entendimiento maimoniano era pensar relaciones entre reglas de producción, la del Yo fichteano se caracterizará esencialmente como un poner, y ante todo, un ponerse a sí mismo. Tal el sentido de la Thathandlung, la actividad originaria, fundamento de toda conciencia, expresada en los tres axiomas del FDC (§ 1-3). Aquí, el Yo es posición absoluta de sí y de toda realidad, acto que encuentra una oposición absoluta en un No-Yo, la cual es conciliada en y por el Yo absoluto, mediante su posición del Yo y No-Yo como instancias relativas, divisibles o mutuamente determinables. Que efectivamente Fichte consideró la teoría maimoniana, se 
ve en un pasaje del § 1 del FDC, donde Fichte afirma el derecho que poseemos de aplicar la categoría de realidad. ${ }^{20}$ Esta categoría, como las demás, se deriva del Yo como sujeto absoluto, cuya realidad no depende ni se deriva de nada superior (y, en este sentido, podríamos pensarla como supra-categorial).

20 En cuanto al modo en que será desplegado el sistema, es preciso ver una novedad del planteo fichteano en la introducción del No-Yo, y el consecuente conflicto que éste genera para el Yo. Esta instancia permitirá articular el aspecto absoluto con el limitado del Yo, estableciendo una serie de mediaciones que permitirán derivar el sistema de las determinaciones de la experiencia efectiva. La oposición absoluta, incondicionada en su forma pero dependiente del Yo (en la medida en que es lo absolutamente opuesto a él), exige por su mera posición la anulación de la autoposición del Yo, y con eso la del propio No-Yo, amenazando la unidad de la conciencia (dato que incluso el escéptico no puede negar). ${ }^{21}$ La conciliación de la contradicción exige entonces una determinación que permita pensar simultáneamente posición y oposición, realidad y negación. Esta determinación está dada por la limitación, ${ }^{22}$ por la cual el Yo, absolutamente puesto y enfrentado al No-Yo absolutamente opuesto, pone en sí un Yo y un No-Yo divisibles, mutuamente determinados, lo que abre el pasaje al desarrollo de la parte teórica de la FDC (§ 4), encargada de agotar, por vía de un método analítico-sintético, el contenido de su proposición principal: "el Yo se pone como determinado por el no-Yo". Esta proposición contiene en sí una antítesis ("El No-Yo determina al Yo" y "El Yo se determina a sí mismo") conciliada sintéticamente por un concepto que será vertebral en el desarrollo de la FDC: la determinación recíproca (Welchselbestimmung). ${ }^{23}$ El esquema de la determinación recíproca, aplicado a las antítesis contenidas en la proposición principal, conduce a las categorías de causalidad (causa-efecto determinados como grados de hacer-padecer en reciprocación) y sustancialidad (sustancia-accidente como modos de una exclusión mutua en reciprocación). ${ }^{24}$

21 La influencia de la ley de la determinabilidad de Maimon sobre Fichte ha sido señalada por Daniel Breazeale. Según este autor, si bien Fichte rechaza la concepción escéptica maimoniana,

abraza sin embargo la idea de la filosofía como un sistema del «pensamiento real», aun cuando transforma la idea de Maimon acerca de cómo debía alcanzarse esa meta -una transformación que involucra otra, del principio de determinabilidad a un nuevo principio de determinación recíproca. ${ }^{25}$

Mientras que Maimon ponía los conceptos relacionales en un lugar secundario respecto al concepto absoluto, dado por la determinabilidad, Fichte hace de la relación la vía para exponer el proceder del Yo absoluto. La característica distintiva del pensamiento real ya no reside (como lo hacía para Maimon) en una síntesis unilateral sino en una síntesis de determinación recíproca. Esto implica una nueva relación entre sujeto y predicado (determinable y determinación), que Fichte desarrolla en su tratamiento del concepto de sustancialidad. Maimon definía la determinabilidad por la unilateralidad y necesidad de la deducción de las determinaciones de un determinable pensado como sujeto de una infinitud de determinaciones, derivables según una regla de producción. En ese marco, los entendimientos finitos caían en síntesis arbitrarias, no fundadas en las reglas de producción de los objetos sino en meras relaciones entre intuiciones. El entendimiento infinito contenía todos los determinables y los determinaba en y por sí mismo. El fundamento de relación entre uno y otro permanecía oculto. En ese sentido, le cabe a Maimon la crítica que Fichte dirige al idealismo dogmático: "En este sistema no se puede indicar fundamento alguno para la limitación de la realidad en el yo (es decir para la 
afección por la cual surge una representación)". ${ }^{26}$ La mera postulación de un entendimiento infinito (se lo conciba como ideal o real) no explica lo que debe ser explicado: la génesis de la limitación que fundamenta la conciencia empírica. Salir del escepticismo maimoniano implica mostrar la conexión entre infinitud y finitud, o mejor, absoluto y limitado.

23 Para ello, veamos en primer lugar cómo Fichte utiliza el término "determinabilidad". Este concepto aparece en el desarrollo de la síntesis de sustancialidad, derivado de la noción de relación. Según se dice allí, "la relación debe ser absoluta, y lo absoluto no debe ser nada más que una relación" ${ }^{27}$ (de hecho, esto se constata desde el fundamento del sistema, donde la posición y oposición absolutas resultan necesariamente en la relación recíproca). En este esquema, la noción de determinabilidad es definida como "la materia de la reciprocación", ${ }^{28}$ siendo la forma de la misma "el mutuo excluir y ser-excluido de los miembros de la reciprocación entre sí". ${ }^{29}$ La posición de un objeto como determinado y completo (por ejemplo, un trozo de hierro) implica concebirlo como una totalidad; pero en la medida en que una nueva determinación, antes excluida de esa totalidad, puede alcanzarla (por ejemplo, el hierro entra en movimiento), la totalidad se abre, un aspecto de ella deviene indeterminado, pero determinable: exige recibir una nueva determinación (por ejemplo, ser atraído por un imán). "Lo determinable debe ser determinado por lo determinado; y lo determinado debe ser determinado por lo determinable; y la unidad que de aquí surge es la totalidad que buscamos". ${ }^{30}$ La totalidad debe quedar abierta o indeterminada en algún aspecto, para ser determinable, y la síntesis de determinado y determinable no se caracteriza por su unilateralidad, sino por su reciprocidad. La totalidad descripta consiste en una determinabilidad determinada, o sustancia. Ahora bien, originariamente, la determinabilidad no es un concepto de los objetos de la experiencia, sino un modo de actuar del Yo, o más precisamente, una tarea que éste se impone a sí mismo: la tarea de poner una determinación por sí mismo. ${ }^{31}$ La posición de un límite, exigida por la reciprocación propia de la determinabilidad, presupone un choque en la actividad originaria del Yo, proyectada al infinito. Este choque, que posibilita la reflexión del Yo sobre sí, es atribuido a un no-Yo. La finitud de la determinabilidad debe estar entonces acompañada por la infinitud o indeterminabilidad de la actividad, que excede todo límite y a la vez sólo se conoce en tanto pone un límite. De ese modo, finitud e infinitud se concilian: "si no hay infinitud no hay limitación; si no hay limitación, no hay infinitud; infinitud y limitación están conciliadas en un solo y mismo miembro sintético" ${ }^{32}$ En la medida en que se plantea como una tarea autoimpuesta, la determinabilidad deja de remitir exclusivamente a una razón cognoscitiva, y remite a una razón práctica. Así lo anticipa Fichte en esta sección: "se verá en la parte práctica que la determinabilidad, de la cual se trata aquí, es un sentimiento. Pero un sentimiento es sin duda una determinación del Yo, pero no del Yo como inteligencia". ${ }^{33}$ Veamos entonces, en segundo lugar, cómo se retoma esta idea en la parte práctica del FDC.

24 El "Fundamento de la ciencia de lo práctico" (\$ 5-11) se basa en un desarrollo de la proposición "El Yo se pone como determinando al no-Yo". Esta proposición "presupone la determinabilidad, por tanto la realidad del no-Yo" ${ }^{34} \mathrm{El}$ método a seguir en la parte práctica implica resolver el conflicto que queda abierto por la parte teórica: aquél que se da entre el Yo puesto como Inteligencia (limitado) y el Yo como absolutamente puesto (ilimitado). El Yo sólo puede ser limitado en tanto inteligencia, como actividad cognoscitiva, y esto implica una relación causal con un no-Yo que produce el mencionado choque en la actividad originaria proyectada al infinito (y que sólo posee realidad transferida por el Yo, en la 
medida en que el choque acontece). Aquí no se trata ya de la relación entre el Yo y el noYo, sino del Yo en determinación recíproca consigo mismo (Yo como inteligencia, limitado, y Yo como actividad pura, absoluto). La noción de sentimiento (a la cual, según vimos, Fichte asociaba la determinabilidad) no se explica por el influjo de una materia extraña sobre el Yo, sino por la limitación del Yo por sí mismo, en virtud de sus propias leyes, en ocasión del factum indemostrable del choque. El sentimiento expresa entonces un constreñimiento de la tendencia a la infinitud, por su limitación (§ 7). Pero dado que el Yo no es consciente de su obrar en esta limitación (la cual es condición de la conciencia), éste atribuye la limitación a una cosa. ${ }^{35}$ Éste es el fundamento del sentimiento de algo $=\mathrm{X}$ determinado independientemente del Yo (pero, precisamente, esta independencia es meramente sentida, dada la inconsciencia del Yo sobre su obrar, de la cual el sentimiento depende). La facultad práctica exige que el Yo se ponga como determinando ese $\mathrm{X}$ que se le manifiesta como independiente de sí. Para ello, Fichte caracterizará la actividad del Yo como un impulso a la determinación recíproca:

Lo que intuye debe limitar X por espontaneidad absoluta y precisamente de tal manera que $\mathrm{X}$ aparezca como limitado únicamente por sí mismo -tal era la exigencia. Ésta es satisfecha cuando la actividad ideal, por su facultad de producción absoluta, pone más allá de $\mathrm{X}$ un $\mathrm{Y}$ [...] $\mathrm{X}$ e $\mathrm{Y}$ deben excluirse mutuamente. En la medida en que, como ocurre aquí, este impulso sólo se orienta a la actividad ideal, podemos llamarlo impulso a la determinación recíproca. [...] El impulso a la determinación recíproca intenta, pues, a la vez un sentimiento. Por consiguiente, en este impulso están estrechamente conciliados la actividad ideal y el sentimiento; en este impulso todo el yo es uno. En esta medida podemos llamarlo impulso proyectado a la reciprocación en general. Este impulso es el que se manifiesta por el anhelo; el objeto del anhelo es algo distinto, algo opuesto a lo existente. ${ }^{36}$

Así, la determinación de la experiencia efectiva dada por un sentimiento actual (X) presupone el trascender el mismo hacia un producto de la actividad ideal (Y), al cual, en tanto se determina como distinto $\mathrm{u}$ opuesto a $\mathrm{X}$, le caben todas las posibles determinaciones, salvo las existentes en X (que, a su vez, es determinado por ellas). La determinación se caracteriza entonces como acto de determinación negativa, pudiendo poner algo opuesto a lo existente que determina a su vez eso existente, y dirigirse luego a la efectuación de lo puesto, acto que puede ser continuado indefinidamente, ${ }^{37} \mathrm{y}$ que admite la posición de diferentes productos ideales no predeterminados (lo cual a su vez resulta en diferentes modos posibles de determinar lo dado).

Esta idea de una continuación indefinida de la determinación nos reenvía a aquélla según la cual todo lo determinado está necesariamente en reciprocación con una esfera indefinida de determinaciones posibles. Esta esfera es puesta sólo por la actividad ilimitada del Yo absoluto, que debe devenir finito, y, en la medida en que lo hace, debe superar esa limitación. Es por la espontaneidad absoluta del Yo, y según las leyes de su desarrollo, que éste pone las determinaciones de la realidad efectiva al enlazarlas en determinación recíproca con la determinación ideal. El Yo se lanza entonces, por su propia dinámica, hacia determinaciones siempre nuevas, cuya fuente es él mismo: es él quien pone la dirección, y se autodetermina a sí mismo a seguirla; y esto se da no en un sentido temporal, sino en la misma unidad estructural de la subjetividad trascendental. El modelo de la progresión infinita de Maimon, a pesar de identificar la producción y la cognición de la realidad con la actividad intelectiva, no logra articular esta actividad en sus aspectos finito e infinito sino privilegiando lo infinito como horizonte pre-establecido sobre el cual lo finito no tiene ninguna incidencia. Aun considerando que este autor caracterizó al entendimiento infinito como un ideal, e incluso postuló una posible salida a 
su propio escepticismo mediante la noción de una constante acción en aras de la aproximación a ese ideal (por lo cual podría decirse que había ya preparado el terreno para la respuesta fichteana), ${ }^{38}$ lo cierto es que sus conclusiones serán siempre llevadas hacia la negación de validez de nuestro conocimiento de la experiencia, y esto a causa de la finitud. La concepción de un entendimiento infinito, que concibe la completitud material de las reglas formales del entendimiento, implica la idea de que la totalidad preexiste de manera completamente determinada, independientemente de la actividad finita. Esta preexistencia implica que el modelo bajo el cual podemos pensar la aproximación al ideal es el de la repetición monótona de una misma operación, negándole capacidad productiva al ser racional finito y condenándolo a permanecer en la esfera del escepticismo. Frente al privilegio maimoniano de la razón cognoscitiva infinita que produce la totalidad de acuerdo a la determinabilidad, Fichte da un rol determinante a la actividad práctica productiva de determinaciones ideales que dan consistencia y sentido a las determinaciones de la experiencia efectiva, en un esquema en que infinitud y finitud quedan conciliadas en una relación de reciprocidad.

27 En lo que precede hemos esbozado un sentido para los conceptos de determinabilidad y relación recíproca en el sistema de Maimon con vistas a esclarecer algunos de problemas que asoman en el horizonte de Fichte durante la elaboración de su propio sistema. Recuperamos la presentación fichteana de la noción de determinabilidad mostrando cómo ésta aparece resignificada desde la noción de determinación recíproca. Intentamos asimismo mostrar cómo esa resignificación, en el marco de una reivindicación ontológica de la finitud, puede leerse como una respuesta al "trascendentalismo escéptico" maimoniano. A diferencia del modelo de la progresión infinita, el Yo fichteano sigue el de la continuación indefinida de la determinación, considerando que todo determinado está necesariamente en reciprocación con una esfera indefinida de determinaciones posibles, puesta idealiter por la espontaneidad del Yo absoluto. Podemos en cada caso continuar indefinidamente siguiendo una dirección autoimpuesta sin necesidad de presuponer un destino previamente fijado con independencia de nuestra propia actividad. En virtud de ello, podemos incluso habilitar ontológicamente la posibilidad de un corte, y el trazado de una nueva dirección, cuya posición implica la génesis de determinaciones nuevas que adquirirán consistencia en el trazado y el recorrido mismos.

28 La progresión infinita, ejemplificada en el modelo del método exhaustivo, supone entonces una meta a la vez previamente dada y fácticamente inalcanzable, lo cual deriva en una perspectiva cognoscitiva y práctica desesperante (por mucho que los fines explícitamente propuestos por Maimon no apunten en esta dirección). Frente al movimiento unilateral, unidireccional e infinito que este modelo supone, creemos que puede leerse en la determinación recíproca fichteana una dialéctica que, lejos de remitir a una permanente negación destructiva de lo dado para afirmar un porvenir eternamente diferido, implica una dinámica compleja entre lo dado y aquello que da sentido a lo dado. Lo dado, en su simpleza improductiva, su finitud y consistencia efectiva, depende esencialmente de un no-dado entrelazado necesariamente con él, elaborado por medio de una espontaneidad absoluta que constituye el ámbito de lo infinito e ideal. En la luz que este último echa retroactivamente sobre el primero se erige un modelo cuyas consecuencias están más próximas al entusiasmo práctico que a la desesperanza 
cognoscitiva. Finitud e infinitud quedan unificadas en un Yo capaz de superar cualquier límite que pueda captar, y que de hecho supera en la medida en que lo capta: razón de que existamos continuamente más allá de nosotros mismos.

\section{Bibliografía} Tecnos: Madrid. philosophy: Satz der Bestimmbarkeit and the role of synthesis", en: FREUDENTHAL, G. (ed.), Salomon Maimon. Rational dogmatic, empircal skeptic, Dordrecht: Klwuer Academic Publishers, 18-53.

$40-101$.

ACOSTA, E., (2015), "La arquitectónica de la deducción de las categorías en el sistema fichteano de 1794/95", Revista de Filosofía Aurora, Curitiba: v. 27, 689-710 (consultado el 20 de julio de 2017, URL: http://www2.pucpr.br/reol/pb/index.php/rf? dd1=15967\&dd99=view\&dd98=pb).

BEISER, F., (2003), "Maimon and Fichte", en: G. FREUdENTHAL (ed.), Salomon Maimon. Rational dogmatic, empircal skeptic, Dordrecht: Klwuer Academic Publishers, 233-248.

BREAZEALE, D., (2003), "Der Satz der Bestimbarkeit: Fichte's Reception and Transformation of Maimon's Principle of Synthetic Thinking", Internationales Jahrbuch des Deutschen Idealismus, $\mathrm{n}^{\circ} 1,115-140$.

FICHTE, J. G., GWL: Grundlage der gesammten Wissenschaftslehre. Citado con la sigla GA según la edición J. G. Fichte-Gesamtausgabe der Bayerischen Akademie der Wissenschaften. R. Lauth et al. (eds.). Stuttgart-Bad Cannstatt, Frommann-Holzboog, 1962 ss., con indicación de volume y paginación

FICHTE, J. G., FDC: trad. J. CRUZ CRUZ, Doctrina de la ciencia, Buenos Aires: Aguilar, 1975.

FICHTE, J. G., FDN: trad. J. VILLACAÑAS, M. RAMOS, y F. oncinA COVES, Fundamento del derecho natural según los principios de la doctrina de la ciencia, Madrid: Centro de estudios constitucionales, 1994.

KANT, (2009), Crítica de la razón pura, trad. M. CAIMI, Buenos Aires: Colihue.

MAIMON, S., (1790a), "Antwort auf voriges Schreiben”, en: Berlinisches Journal für Aufklärung, IX (incluído en Versuch über die Transzendentalphilosophie, Hamburgo: Felix Meiner Verlag).

MAIMON, S., (2004 [1790b]), Versuch über die Transzendentalphilosophie, Hamburgo: Felix Meiner Verlag.

ONCINA COVES, F., (1989), "Maimon y Fichte. Una interpretación poskantiana de la filosofía práctica del criticismo", en muguerzA, J. Y ARAmayo, R. R. (eds.), Kant después de Kant,

SCHECHTER, O., (2003), "The logic of speculative philosophy and skepticism in Maimon's 


\section{NOTAS}

1. GA, III/2, 282 (carta de Fichte a Reinhold de marzo de 1795). Tomamos la referencia y traducción de oncina Coves, F. 1989: 399.

2. GWL, GA I/2, 368 / FDC, 99. Al citar esta obra, seguimos en todos los casos la traducción de J. Cruz Cruz, indicando su número de página con las siglas "FDC" a continuación de la referencia a la GA.

3. Ver Oncina Coves, F. 1989: 392 y ss.

4. Maimon 1790a: 52, yo traduzco.

5. Maimon 1790a: 52, cursivas mías.

6. Maimon 1790b: 40, las traducciones de las referencias a esta obra son mías.

7. Diferencia que queda ilustrada, por ejemplo, en el célebre pasaje de la Crítica de la razón pura en el que Kant remite a la sensibilidad y el entendimiento como los dos "troncos del conocimiento humano" que, si bien son heterogéneos, "quizá broten de una raíz común, aunque desconocida para nosotros" (KrV A 15 - B 29; en todos los casos en que refiramos a esta obra seguiremos la traducción española de Mario Caimi, cuya paginación aclararemos a continuación del siguiente modo: Kant 2009: 89). Contra la postura gnoseológica de Kant, que vedaba el acceso del pensamiento humano a esa posible "raíz común", todo el esfuerzo del pensamiento de Maimon se dirige a internarse en ella, bajo la convicción de que sólo desde su perspectiva los conocimientos son firmes y seguros.

8. Maimon 1790b: 23.

9. "Cuando una percepción, por ejemplo el rojo, me es dada, no tengo ninguna conciencia de ella; cuando otra, por ejemplo verde, me es dada, no tengo tampoco conciencia de ella. Pero si relaciono una con otra (a través de la unidad de la diferencia), descubro que el rojo es diferente del verde, y obtengo conciencia de cada una de las percepciones en sí mismas." (Maimon 1790b: 77).

10. Maimon 1790b: 52 (nota al pie), cursivas mías.

11. Para un recorrido exhaustivo sobre este concepto en la obra maimoniana, ver el trabajo (que seguimos aquí) de Schechter 2003: 18-53.

12. Maimon, 1790b: 73, cursivas mías.

13. Maimon 1790b: 44-45.

14. Ver los primeros párrafos de la introducción del EFT, donde la pluralidad de impulsos [Triebs] humanos aparecen como pasibles de reducirse a un único impulso a pensar, tendiendo continuamente a un máximum que no puede rebasarse, por estar este impulso limitado subjetivamente, aunque no objetivamente (Maimon 1790b: 7).

15. Por ejemplo, en "La antinomia de la razón pura", donde Kant habla de la muerte de la filosofía cuando ella se deja llevar ya por la desesperanza escéptica, ya por el empecinamiento dogmático (ver KrV A 407 - B 434 / Kant 2009: 489).

16. GNR, GA I/3, p. 318 (nota). Seguimos la traducción castellana de Villacañas, J., Ramos, M., y Oncina, F. (FDN, 107)

17. BWL, GA I/2, p. 135, yo traduzco.

18. BWL, GA I/2, p. 135.

19. "El gran éxito que la razón obtiene por medio de la matemática conduce naturalmente a presumir que, si no ella misma, [al menos] el método de ella tendrá buen éxito también fuera del campo de las cantidades, ya que él reduce todos sus conceptos a intuiciones que puede dar a priori , por medio de lo cual llega a dominar, por decirlo así, a la naturaleza; mientras que la filosofía 
pura, por el contrario, chapucea aquí y allá en la naturaleza con conceptos discursivos a priori, sin poder tornar intuitiva a priori la naturaleza de ellos, y sin poder tornarla, precisamente por este medio, fidedigna" (KrV A 724-5 - B 752-3 / Kant 2009: 754-5). Si bien esta crítica se vincula a la aplicabilidad filosófica del método geométrico a la Spinoza (y en ese sentido podría dirigirse también a ciertos aspectos de la construcción fichteana), nos interesa recuperar la distinción tajante que tanto Kant como Fichte establecen a este respecto entre ambas disciplinas. Maimon posee su propio criterio de división, relativa a la relación de la matemática a la intuición a priori y de la filosofía a la intelección pura, el cual no lo incomoda a la hora de extrapolar figuras de una a la otra.

20. Ver GWL, GA I/2, 262 / FDC, 19. Fichte se refiere a una obra de Maimon más cercana cronológicamente a la GWL, Incursiones en el ámbito de la filosofía. Allí, Maimon desarrolla su postura (que caracterizamos en la sección anterior) según la cual no estamos en condiciones de demostrar la realidad de nuestros juicios de la experiencia.

21. Sobre esta serie de contradicciones por las cuales los dos primeros principios se anulan mutuamente a sí mismos, ver GWL, GA I/2, 268-9 / FDC, 24.

22. Ver GWL, GA I/2, 270 / FDC, 25.

23. GWL, GA I/2, 290 / FDC, 42.

24. Sobre la fundamental importancia del concepto de determinación recíproca para toda la deducción fichteana de las categorías, y un pormenorizado análisis de la estructura de esta deducción, ver Acosta 2015: 689 y ss.). En particular, sobre la síntesis de sustancialidad, el autor afirma: "Realidad y negación son, pues, deducidas como determinabilidad y ser determinado mediante relativización del par conceptual hacer/padecer" (Acosta 2015: 702, cursivas mías), mostrando así la noción de determinabilidad como dependiente de la de determinación recíproca, idea que retomaremos en breve.

25. Breazeale 2003: 116, traducción mía. Si bien este autor traza un importante recorrido historiográfico relativo a la influencia maimoniana en la producción del Fichte del FDC y la Nova methodo, creemos que su tratamiento del tema es insuficiente en tanto no desarrolla el lugar que la noción de determinabilidad tiene en la FDC, ni el modo en que la determinación recíproca se hace presente en la parte práctica de dicha obra. A estos temas se dedica lo que resta del presente trabajo.

26. GWL, GA I/2, 310 / FDC, 57.

27. GWL, GA I/2, 345 / FDC, 83.

28. GWL, GA I/2, 343 / FDC, 81.

29. GWL, GA I/2, 342 / FDC, 81.

30. GWL, GA I/2, 346 / FDC, 83.

31. Ver GWL, GA I/2, 355 / FDC, 89.

32. GWL, GA I/2, 358 / FDC, 91.

33. GWL, GA I/2, 358 / FDC, 91, cursivas mías.

34. GWL, GA I/2, 386 / FDC, 115.

35. Ver GWL, GA I/2, 441 / FDC, 159.

36. GWL, GA I/2, 443-4 / FDC, 160-1.

37. Ver GWL, GA I/2, 445 / FDC, 162.

38. Así lo sostiene, por ejemplo, Frederick Beiser. Ver Beiser 2003: 245. 


\section{RESÚMENES}

The aim of this paper is to inquire into a possible implicit influence of the Essay on Transcendental Philosophy (1790) of Salomon Maimon on the Foundations of the Science of Knowledge (1794/95) of Johann G. Fichte. On the one hand, Fichte gives great importance to Maimon's criticism towards the kantian separation between phenomena and thing-in-itself; on the other hand, Maimon's skeptical challenge is a position against which Fichte reacts in his '94/95 work.

In order to determine this influence we will focus in two specific concepts which are present in both thinker's systems: the laws of determinability and of reciprocal determination. While Maimon grants a privilege to the first of these concepts to explain the transcendental synthesis of real thinking, Fichte favors the second one. The result is a divergence between both systems regarding their point of view on the structure of subjectivity. We will argue that Maimon remains on the cognitional reason viewpoint, from where he sees subjectivity as divided in a finite and an infinite understanding. On the contrary, Fichte's perspective is that of practical reason, where the conflict between finite and infinite is internalized in the production of every single real determination.

\section{ÍNDICE}

Keywords: subjectivity, law of determinability, reciprocal determination, finite-infinite, Maimon

\section{AUTOR}

GONZALO SANTAYA

CONICET/Universidad de Buenos Aires 